\title{
A Retrospective, Cross-Sectional Study on the Prevalence of Hyperuricemia Using a Japanese Healthcare Database
}

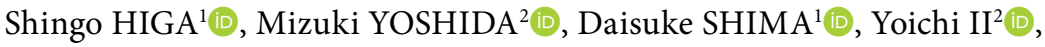 \\ Shigeru KITAZAKI ${ }^{3}$, Yuji YAMAMOTO ${ }^{3}$, Yoko FUJIMOTO' ${ }^{10}$ \\ ${ }^{1}$ Pfizer Japan Inc., Medical Affairs, Pfizer Essential Health, Tokyo, Japan \\ ${ }^{2}$ Pfizer Japan Inc., Clinical Statistics, Development Japan, Tokyo, Japan \\ ${ }^{3}$ Minacare Co., Ltd., Tokyo, Japan
}

\begin{abstract}
Objectives: This study aims to evaluate the prevalence of hyperuricemia (HU) considering both serum uric acid (SUA) levels and medication status of urate-lowering drugs (ULDs), and the association between $\mathrm{HU}$ and its comorbidities using a Japanese healthcare database.

Materials and Methods: The study population consisted of 60,828 subjects who had at least one serum uric acid measurement between the fiscal years (FYs) 2010 and 2014 in a Japanese employment-based health insurance database (MinaCare Co., Ltd., Tokyo, Japan), which includes mutually linked medical/pharmaceutical claims data and health check-up data. Hyperuricemia was defined as a SUA level $>7.0 \mathrm{mg} / \mathrm{dL}$ of the health check-up data and/or a prescription for a ULD. The association between HU and comorbidities were analyzed by comparing the prevalence of HU of each subgroup defined by presence or absence of comorbidity.

Results: The prevalence of HU in FY 2014 was $26.8 \%$ (95\% confidence interval [CI]: 26.2 to $27.3 \%$ ) in male subjects and $0.9 \%$ ( $95 \%$ Cl: 0.7 to $1.0 \%$ ) in female subjects. According to the analyses by sex and age, a trend of increasing prevalence with age was observed in both males and females. The prevalence of $\mathrm{HU}$ remained stable both in males and females from FYs 2010 to 2014. The positive association between HU and well-known comorbidities were confirmed with the exception of diabetes mellitus and smoking status in male subjects.

Conclusion: Our results provided a more accurate prevalence of $\mathrm{HU}$ in Japanese population. It is important to increase the awareness on $\mathrm{HU}$ in the society to reduce the burden of HU-related diseases.

Keywords: Claims data, hyperuricemia, MinaCare database, prevalence.
\end{abstract}

Hyperuricemia (HU) is an important pathological condition that is not only a direct cause of gout, ${ }^{1}$ but also an independent risk factor for the development of hypertension (HT), ${ }^{2,3}$ chronic kidney disease (CKD), ${ }^{4}$ and end-stage kidney disease. ${ }^{5}$ In addition, accumulated evidence suggests that hyperuricemia may have a pathogenic role in the development of metabolic syndrome, ${ }^{6}$ which is defined as a cluster of cardiovascular risk factors such as elevated glucose level, central obesity, HT, hypertriglyceridemia and low high-density lipoprotein cholesterol. ${ }^{7}$ There are many unknown aspects in this area; for example, the etiological mechanisms of high blood pressure and renal damage caused by HU have not been clearly elucidated, and the causal relationship between HU and cardiovascular events is still controversial. The accurate prevalence of $\mathrm{HU}$ in Japan is also one of the unknown aspects.

Received: December 03, 2018 Accepted: March 27, 2019 Published online: April 22, 2019

Correspondence: Yoko Fujimoto. Pfizer Japan Inc., Medical Affairs, Pfizer Essential Health, 1518589 Tokyo, Japan. Tel: +81-80-6809-9575 e-mail: yoko.fujimoto@pfizer.com 
A few studies have reported the prevalence of $\mathrm{HU}$ in the Japanese population on the basis of the results of health check-ups in certain populations. ${ }^{8,9}$ A study was performed at a health screening center and the results indicated that the prevalence of $\mathrm{HU}$ in males was $21.5 \%$ as of $2003 .{ }^{8}$ Another population-based study conducted in Northern Japan reported a HU prevalence of $17.4 \%$ in male subjects and $2.2 \%$ in female subjects. ${ }^{9}$ However, the estimations of $\mathrm{HU}$ prevalence in these studies did not consider the number of well-managed $\mathrm{HU}$ patients on urate-lowering drugs (ULDs), and are therefore presumed to underestimate the actual figures.

Previously reported age distributions of $\mathrm{HU}$ patients also imply underestimation of the $\mathrm{HU}$ prevalence. It was reported that the prevalence of $\mathrm{HU}$ increases with age in the USA and China, ${ }^{10,11}$ whereas epidemiological studies conducted in Japan reported a higher prevalence of $\mathrm{HU}$ in male subjects in their 30 s and 40 s than in older age groups. ${ }^{8,12}$ This age distribution of HU patients in Japan is considered to reflect the fact that well-controlled HU patients on ULDs are excluded, because $\mathrm{HU}$ is included in the indication of ULDs in Japan unlike in other countries. Similar considerations are also mentioned in the Japanese guideline for the management of $\mathrm{HU}$ and gout. ${ }^{13}$ Therefore, in this study, we aimed to evaluate the prevalence of $\mathrm{HU}$ considering both serum uric acid (SUA) levels and medication status of ULDs, and the association between $\mathrm{HU}$ and its comorbidities using a Japanese healthcare database.

\section{MATERIALS AND METHODS}

This was a retrospective, cross-sectional study conducted using a Japanese healthcare database (MinaCare Co., Ltd., Tokyo, Japan). The primary objective of this study was to estimate the prevalence of $\mathrm{HU}$ in Japan considering both SUA levels evaluated as health check-up and medication status of ULDs, and to investigate the trend of $\mathrm{HU}$ prevalence in recent years (fiscal year [FY] 2010 to FY 2014 [FY=April $1^{\text {st }}$ to March 31 ${ }^{\text {st }}$ ).

The secondary objectives of this study were to examine the association of body mass index (BMI), smoking status, and well-known comorbidities of
HU such as HT, diabetes mellitus (DM), and hyperlipidemia (HL) with the prevalence of $\mathrm{HU}$.

The Japanese healthcare database that is managed by MinaCare Co., Ltd. was used for this study. The MinaCare database includes annual health check-up and medical/pharmaceutical claims data that are provided by employmentbased health insurance societies. ${ }^{14}$ The health check-up data include information on subjects' demographics, smoking status, blood pressure, $\mathrm{BMI}$, and clinical laboratory test results. The medical/pharmaceutical claims data include information on prescriptions, medical procedures, diagnosis, and administrative data. Since these two types of data are linked in the subpopulation, this database enables us to estimate the prevalence of HU considering both the SUA levels at health check-ups and the prescriptions for ULDs in the claims data. The populations covered by this database comprise working individuals and their dependent family members. Since the employment-based insurance covers various large-scale, nationwide industries, this database includes healthcare information with minimal geographic or occupational bias.

The MinaCare database used for this research includes only the information that has already been anonymized unlinkably. Approval for this research by an ethical review committee and informed consent of each subject were not required, because studies using only unlinkably anonymized data are outside the scope of "Ethical Guidelines for Medical and Health Research Involving Human Subjects ${ }^{15}$ " set by the Japanese government.

The data that support the findings of this study are available from MinaCare Co., Ltd., but restrictions apply to the availability of these data, which were used under license of the current study, and are not publicly available. Data are however available from the authors upon reasonable request and with the permission of MinaCare Co., Ltd.

The study population consisted of 60,828 subjects who had at least one SUA measurement in their health check-up data during the 2010 to 2014 FYs period. For analysis of a particular FY, subjects with missing SUA values were excluded from the analysis. For subjects with multiple observations of SUA in the same FY, the data of 
the examination date with the highest SUA value were used. Hyperuricemia was defined as a SUA level $>7.0 \mathrm{mg} / \mathrm{dL}$ in the health check-up data and/or a prescription for ULDs (benzbromarone, probenecid, bucolome, allopurinol, febuxostat, or topiroxostat) in the claims data.

\section{Statistical analysis}

The data analysis was conducted based on the study population. The characteristics of the study population as well as those by sex were descriptively summarized. As the primary analysis, the prevalence of HU by sex, and both sex and age was estimated for each FY (from FY 2010 to FY 2014). The prevalence was calculated as the number of subjects who met the definition of $\mathrm{HU}$ (described in the section of study population and case definition) divided by the total number of subjects who had at least one SUA measurement in each FY. The exact two-sided 95\% confidence interval (CI) for the prevalence was calculated using the Clopper-Pearson method. ${ }^{16}$

As the secondary analysis, subgroup analyses of the prevalence of $\mathrm{HU}$ by the well-known comorbidities of HU (presence/absence of HT, $\mathrm{DM}$, or $\mathrm{HL}), \mathrm{BMI}(<18.5,18.5$ to $<25,25$ to $<30$, and $\geq 30$ ), and smoking status (yes, no) were performed. For subgroup analysis, comorbidities such as HT, DM, and HL were defined by prescriptions for each therapeutic medication (anti-hypertensive drugs, anti-diabetic drugs, and anti-hyperlipidemic drugs, respectively). Statistical comparison based on the prevalence of $\mathrm{HU}$ was conducted between categories for each subgroup using the Chi-square test (for HT, DM, HL, and smoking status) and the Cochran-Armitage trend test (for BMI) at the two-sided significance level of 0.05 .

To support the primary analysis, exploratory analyses were performed by changing the definition of prevalence as follows:

- The prevalence of subjects who had SUA measurements exceeding $7.0 \mathrm{mg} / \mathrm{dL}$ at a health check-up (exploratory analysis 1)

- The prevalence of subjects to whom ULDs were prescribed (exploratory analysis 2)

In addition, tumor lysis syndrome, which is caused by the death of cancer cells during cancer treatment, causes a high SUA level. ${ }^{17}$ Therefore, analysis that excluded cancer patients from the study population was also performed as a sensitivity analysis to evaluate the possibility of

All subjects included in MinaCare database during April 1, 2010 and March 31, 2015 ( $\mathrm{n}=3,773,655)$

Excluded:

- Subjects without health check-up data $(n=2,876,183)$

Subjects with health check-up data during

April, 1, 2010 and March 31, 2015 (n=897,472)

Study population (total)

Subjects with serum uric acid measurement in health check-up data during April 1, 2010 and

March 31, $2015(\mathrm{n}=60,828)$

- Fiscal year 2010

$\mathrm{n}=1,208$

- Fiscal year 2011

$\mathrm{n}=22,832$

- Fiscal year 2012

$\mathrm{n}=27,692$

- Fiscal year 2013

$\mathrm{n}=32,095$

- Fiscal year 2014

$\mathrm{n}=49,286$

Figure 1. Flowchart of study population.

Study population was extracted from subjects included in MinaCare database at any time during study period (01 April 2010 - 31 March 2015). 
overestimation caused by subjects using ULDs for tumor lysis syndrome. All analyses were conducted using SAS version 9.4 software (SAS Institute, Cary, NC, USA).

\section{RESULTS}

Of the 3,773,655 subjects included in MinaCare database, 897,472 subjects had at least one health check-up data during the study period (from FY 2010 to FY 2014). Among them, a total of 60,828 subjects had at least one SUA measurement and were included in the analysis. A flowchart of the study population is presented in Figure 1. The numbers of subjects of each FY were 1,208, 22,832, 27,692, 32,095, and 49,286 in FYs 2010, 2011, 2012, 2013, and 2014, respectively. The characteristics of the study population in FY 2014 are shown in Table 1. A total of 49,286 subjects who had at least one SUA measurement in FY 2014 were analyzed. Of the 49,286 subjects, $51.7 \%$ were males and $48.3 \%$ were females. The study population mainly consisted of subjects in their $30 \mathrm{~s}, 40 \mathrm{~s}$, and 50s. The proportion of male subjects with comorbidities, obesity (defined as BMI $\geq 25$ ), and smoking habit was higher than that of female subjects. There were no big differences in the distribution of age, BMI, or smoking status between this study population, which consists of those who had at least one SUA measurement, and the overall population with any health check-up data in the MinaCare database. ${ }^{14}$

Table 1. Characteristics of study population in fiscal year 2014

\begin{tabular}{|c|c|c|c|c|c|c|}
\hline & \multicolumn{2}{|c|}{ Total $(n=49,286)$} & \multicolumn{2}{|c|}{ Male $(n=25,467)$} & \multicolumn{2}{|c|}{ Female $(n=23,819)$} \\
\hline & $\mathrm{n}$ & $\%$ & $\mathrm{n}$ & $\%$ & $\mathrm{n}$ & $\%$ \\
\hline \multicolumn{7}{|l|}{ Age (year) } \\
\hline$<20$ & 91 & 0.2 & 38 & 0.1 & 53 & 0.2 \\
\hline 20 to 29 & 7422 & 15.1 & 2542 & 10.0 & 4880 & 20.5 \\
\hline 30 to 39 & 12155 & 24.7 & 5530 & 21.7 & 6625 & 27.8 \\
\hline 40 to 49 & 15305 & 31.1 & 8229 & 32.3 & 7076 & 29.7 \\
\hline 50 to 59 & 10742 & 21.8 & 6523 & 25.6 & 4219 & 17.7 \\
\hline 60 to 64 & 3018 & 6.1 & 2193 & 8.6 & 825 & 3.5 \\
\hline$\geq 65$ & 553 & 1.1 & 412 & 1.6 & 141 & 0.6 \\
\hline \multicolumn{7}{|l|}{ Comorbidities } \\
\hline HT (anti-hypertensive drugs* use) & 4870 & 9.9 & 3717 & 14.6 & 1153 & 4.8 \\
\hline DM (anti-diabetic drugs** use) & 1307 & 2.7 & 1069 & 4.2 & 238 & 1.0 \\
\hline HL (anti-hyperlipidemic drugs ${ }^{* * *}$ use) & 3460 & 7.0 & 2488 & 9.8 & 972 & 4.1 \\
\hline \multicolumn{7}{|l|}{$\operatorname{BMI}\left(\mathrm{kg} / \mathrm{m}^{2}\right)$} \\
\hline$<18.5$ & 5454 & 11.1 & 647 & 2.5 & 4807 & 20.2 \\
\hline 18.5 to $<25$ & 33994 & 69.0 & 17353 & 68.1 & 16641 & 69.9 \\
\hline 25 to $<30$ & 8201 & 16.6 & 6334 & 24.9 & 1867 & 7.8 \\
\hline$\geq 30$ & 1603 & 3.3 & 1132 & 4.4 & 471 & 2.0 \\
\hline Unknown & 34 & 0.1 & 1 & 0.0 & 33 & 0.1 \\
\hline \multicolumn{7}{|l|}{ Smoking status } \\
\hline Yes & 9732 & 19.7 & 7812 & 30.7 & 1920 & 8.1 \\
\hline No & 38180 & 77.5 & 17036 & 66.9 & 21144 & 88.8 \\
\hline Unknown & 1374 & 2.8 & 619 & 2.4 & 755 & 3.2 \\
\hline
\end{tabular}

HT: Hypertension; DM: Diabetes mellitus; HL: Hyperlipidemia; BMI: Body mass index; * Anti-hypertensive drugs include diuretics, calcium blockers, angiotensin-converting enzyme inhibitors, angiotensin receptor blockers, direct renin inhibitors, selective aldosterone receptor antagonists, alpha blockers, alpha-2 agonists, reserpine, beta blockers, hydralazine, and sodium nitroprusside; ** Anti-diabetic drugs include insulin, glucagon-like peptide-1 receptor agonists, sulfonylureas, biguanides, pioglitazone, alpha-glucosidase inhibitors, glinides, dipeptidyl peptidase-4 inhibitors, and sodium-glucose co-transporter 2 inhibitors; **** Anti-hyperlipidemic drugs include statins, fibrates, ezetimibe, nicotinic acid, ion-exchange resin, probucol, gamma oryzanol, dextran sulfate sodium, polyene phosphatidylcholine, elastase, ethyl icosapentate, and omega-3 acid ethyl esters. 


\begin{tabular}{|lcccc|}
\hline \multicolumn{4}{|l}{ Table 2. Sex- and age-specific prevalence of hyperuricemia in } & fiscal year 2014 \\
\hline Sex & Age (years) & Prevalence & $\%$ & $95 \%$ CI \\
\hline Male & $<30$ & $510 / 2580$ & 19.8 & 18.2 to 21.4 \\
& 30 to $<40$ & $1390 / 5530$ & 25.1 & 24.0 to 26.3 \\
& 40 to $<50$ & $2232 / 8229$ & 27.1 & 26.2 to 28.1 \\
& 50 to $<60$ & $1917 / 6523$ & 29.4 & 28.3 to 30.5 \\
& 60 to $<65$ & $648 / 2193$ & 29.5 & 27.6 to 31.5 \\
& $\geq 65$ & $126 / 412$ & 30.6 & 26.2 to 35.3 \\
Female & Total & $6823 / 25467$ & 26.8 & 26.2 to 27.3 \\
& $<30$ & $8 / 4933$ & 0.2 & 0.1 to 0.3 \\
& 30 to $<40$ & $38 / 6625$ & 0.6 & 0.4 to 0.8 \\
& 40 to $<50$ & $66 / 7076$ & 0.9 & 0.7 to 1.2 \\
& 50 to $<60$ & $73 / 4219$ & 1.7 & 1.4 to 2.2 \\
& 60 to $<65$ & $13 / 825$ & 1.6 & 0.8 to 2.7 \\
$\geq 65$ & $5 / 141$ & 3.5 & 1.2 to 8.1 \\
& Total & $203 / 23819$ & 0.9 & 0.7 to 1.0 \\
\hline CI: Confidence interval. & & & & \\
\hline
\end{tabular}

The prevalence of $\mathrm{HU}$ in FY 2014 was 26.8\% (95\% CI: 26.2 to $27.3 \%$ ) in male subjects and 0.9\% (95\% CI: 0.7 to 1.0\%) in female subjects (Table 2). According to the analyses by sex and age, the prevalence of $\mathrm{HU}$ increased with age in both male and female subjects (Table 2).

The proportion of subjects who had at least one SUA measurement that exceeded $7.0 \mathrm{mg} / \mathrm{dL}$ in FY 2014 was 22.6\% (95\% CI: 22.1 to 23.1\%) in males and $0.8 \%(95 \% \mathrm{CI}: 0.7$ to $0.9 \%)$ in females. The proportion of subjects whose SUA levels exceeded $7.0 \mathrm{mg} / \mathrm{dL}$ was the highest in males in their 30s (exploratory analysis 1, data not shown). On the other hand, the prevalence of HU considering both SUA levels and medication status was the highest in subjects aged 65 years and older (Table 2). The proportion of subjects to whom ULDs were prescribed in FY 2014 was

Table 3. Sex- and age-specific proportion of subjects to whom urate-lowering drugs were prescribed in fiscal year 2014 (exploratory analysis 2)

\begin{tabular}{lcccc}
\hline Sex & Age (years) & Proportion & $\%$ & $95 \% \mathrm{CI}$ \\
\hline Male & $<30$ & $21 / 2580$ & 0.8 & 0.5 to 1.2 \\
& 30 to $<40$ & $118 / 5530$ & 2.1 & 1.8 to 2.5 \\
& 40 to $<50$ & $483 / 8229$ & 5.9 & 5.4 to 6.4 \\
& 50 to $<60$ & $718 / 6523$ & 11.0 & 10.3 to 11.8 \\
& 60 to $<65$ & $311 / 2193$ & 14.2 & 12.7 to 15.7 \\
& $\geq 65$ & $60 / 412$ & 14.6 & 11.3 to 18.3 \\
& Total & $1711 / 25467$ & 6.7 & 6.4 to 7.0 \\
Female & $<30$ & $0 / 4933$ & 0 & - \\
& 30 to $<40$ & $3 / 6625$ & 0.0 & 0.0 to 0.1 \\
& 40 to $<50$ & $7 / 7076$ & 0.1 & 0.0 to 0.2 \\
& 50 to $<60$ & $12 / 4219$ & 0.3 & 0.1 to 0.5 \\
& 60 to $<65$ & $3 / 825$ & 0.4 & 0.1 to 1.1 \\
& $\geq 65$ & $1 / 141$ & 0.7 & 0.0 to 3.9 \\
& Total & $26 / 23819$ & 0.1 & 0.1 to 0.2 \\
\hline CI: Confidence interval. & & & & \\
\hline
\end{tabular}


6.7\% (95\% CI: 7.8 to $8.4 \%$ ) in males and $0.1 \%$ (95\% CI: 0.1 to $0.2 \%$ ) in females. The proportion of subjects to whom ULDs were prescribed was the highest in elderly persons, for both males and females (exploratory analysis 2 , the results are shown in Table 3).

In the sensitivity analysis in which cancer patients were excluded from the study population, the prevalence of $\mathrm{HU}$ in FY 2014 was 26.0\% (95\% CI: 25.3 to $26.6 \%)$ in male subjects and $0.7 \%$ (95\% CI: 0.6 to $0.9 \%$ ) in female subjects (data not shown). There were no big differences between the results of the main analysis and the sensitivity analysis.

The five-year trend in the prevalence of $\mathrm{HU}$ by sex is shown in Figure 2a, and that by sex and age is shown in Figure 2b and 2c. From FY 2010 to FY 2014, the prevalence of $\mathrm{HU}$ remained stable both in male and female subjects (Figure 2a). In the subgroup analysis by sex and age, there were no big changes in the prevalence of $\mathrm{HU}$ over the course of five years (Figure $2 \mathrm{~b}$ and $2 \mathrm{c}$ ).

The prevalence of $\mathrm{HU}$ in the subgroups defined by well-known comorbidities (presence/absence of HT, DM, or HL), smoking habit (yes, no), and that defined by the BMI value $(<18.5,18.5$ to $<25,25$ to $<30, \geq 30)$ was estimated. The results of these subgroup analyses in male and female subjects in FY 2014 are shown in Figure $3 a$ and $3 b$, respectively. In male subjects, the prevalence of $\mathrm{HU}$ in those with HT or HL was higher than in those without these respective conditions $(p<0.00001$ and $p<0.00001$, respectively). There was an increasing trend in the prevalence of $\mathrm{HU}$ along with the BMI value in male subjects $(p<0.00001)$. However, there was no significant difference in the prevalence of $\mathrm{HU}$ between those with and without DM $(p=0.189)$. The prevalence of $\mathrm{HU}$ in those with a smoking habit was lower than in those without a smoking habit $(p=0.00001)$ (Figure 3a).

In female subjects, the prevalence of $\mathrm{HU}$ in those with HT, DM, HL, or those with a smoking habit was higher than in those without the above conditions $(p<0.00001, p<0.00001, p<0.00001$, and $p<0.00001$, respectively). There was also an increasing trend in the prevalence of $\mathrm{HU}$ with increasing BMI in female subjects $(p<0.00001)$ (Figure 3b).

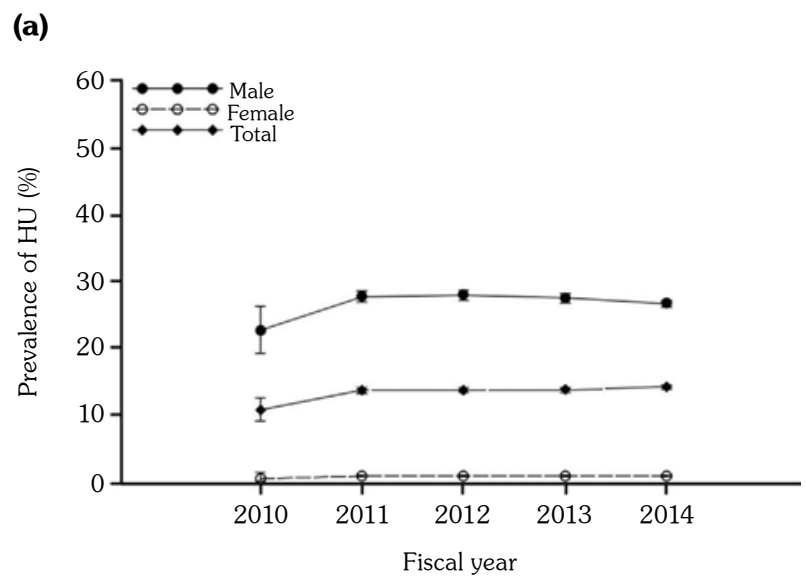

(b)

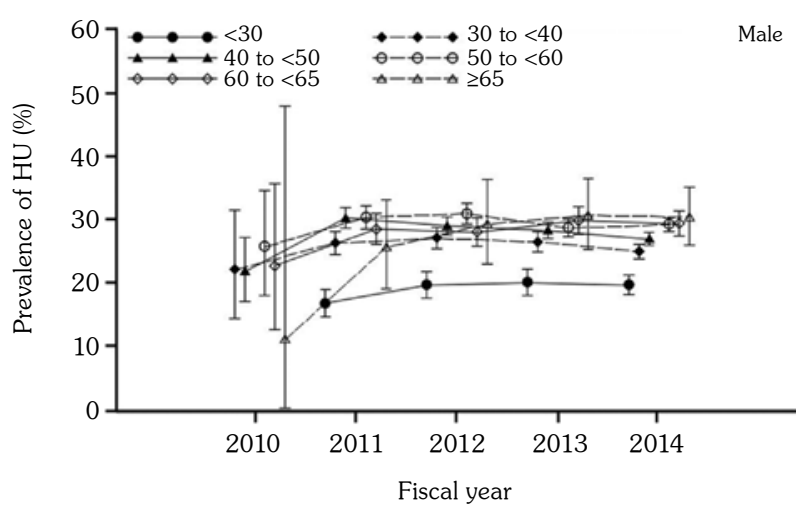

(c)

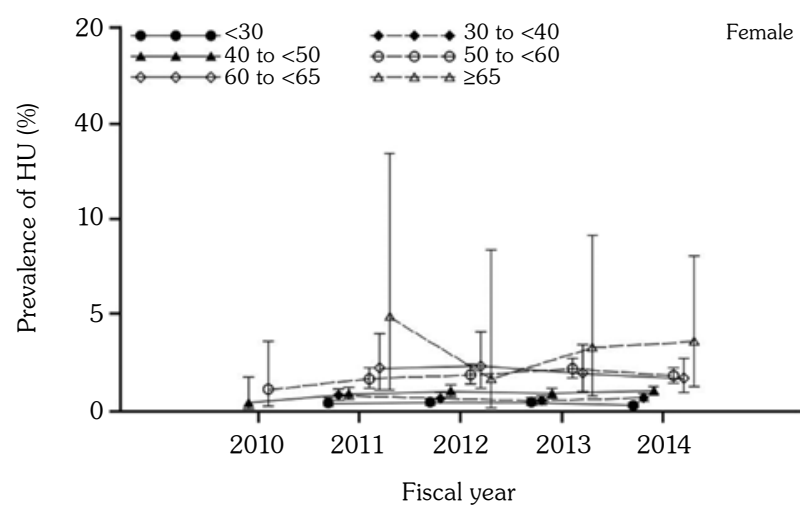

Figure 2. Five-year trend in prevalence of hyperuricemia. (a) Overall and sex-specific prevalence of HU (FY 2010 to FY 2014). (b) Age-specific prevalence of $\mathrm{HU}$ in male subjects (FY 2010 to FY 2014). (c) Age-specific prevalence of $\mathrm{HU}$ in female subjects (FY 2010 to FY 2014). 95\% confidence intervals (shown with bars) were calculated using Clopper-Pearson method.

HU: Hyperuricemia; FY: Fiscal year. 
(a)

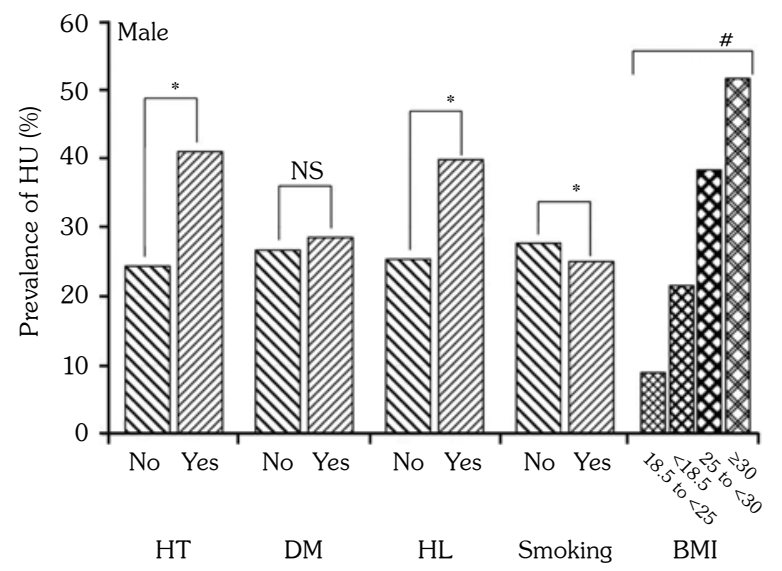

(b)

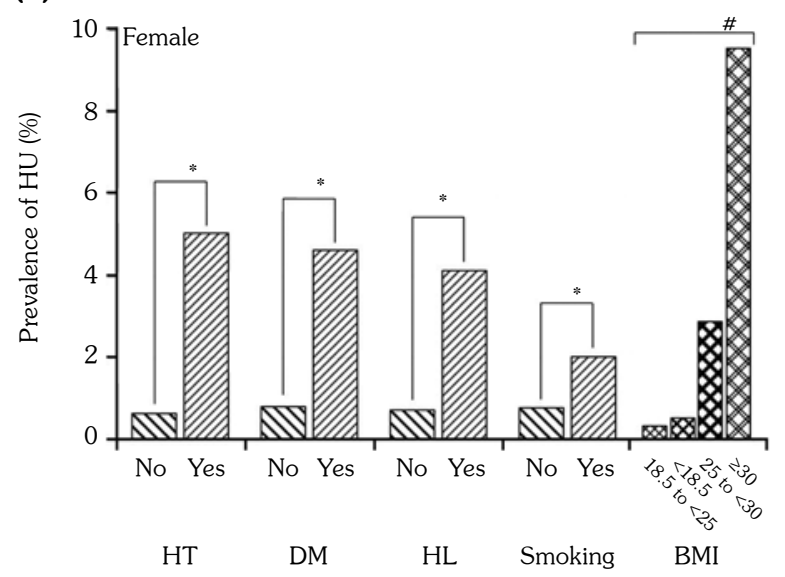

Figure 3. Prevalence of hyperuricemia by major comorbidities of hyperuricemia. Prevalence of hyperuricemia in subgroup defined by presence or absence of comorbidities (hypertension, diabetes mellitus, and hyperlipidemia), smoking habit, and that defined by body mass index value in (a) male subjects and (b) female subjects in fiscal year 2014.

${ }^{*}$ p $<0.0001$ (Chi-square test); \# p<0.0001 (Cochran-Armitage trend test); NS: Not significant; HU: Hyperuricemia; HT: Hypertension; DM: Diabetes mellitus; HL: Hyperlipidemia; BMI: Body mass index.

\section{DISCUSSION}

This study was designed to estimate the prevalence of $\mathrm{HU}$ in the Japanese population more accurately by using data on SUA levels as well as medication status. The MinaCare database, which includes linked health check-up data and claims data, enabled us to estimate the prevalence that also covers well-managed $\mathrm{HU}$ patients. Considering the existence of $\mathrm{HU}$ patients whose SUA levels were controlled at $7.0 \mathrm{mg} / \mathrm{dL}$ or less with ULDs, the prevalence of $\mathrm{HU}$ in FY 2014 in Japanese male subjects observed in this study $(26.8 \%)$ was higher than that previously reported $(21.5 \%, 17.4 \%),{ }^{8,9}$ which is in alignment with our hypothesis. In the analysis using only the SUA level (exploratory analysis 1, data not shown), the overall prevalence and age specific prevalence in male subjects in FY 2014 were very similar to that in a previous study, ${ }^{8}$ in which prevalence of $\mathrm{HU}$ was evaluated based only on the SUA level. In addition, the prevalence of male subjects to whom ULDs were prescribed increased dramatically with age (exploratory analysis 2, Table 3). These data indicate that the previously reported prevalence of $\mathrm{HU}$ based only on the SUA levels were underestimated, particularly in older male patients, as stated in the Japanese guideline for the management of hyperuricemia and gout. ${ }^{13}$ Given the previous study results showing a relationship between the prevalence of gout and aging in several countries ${ }^{18}$ and a relationship between the prevalence of $\mathrm{HU}$ and aging in the USA ${ }^{10}$ and China, ${ }^{11}$ where $\mathrm{HU}$ is not an indication for ULDs, the results observed in this study are considered reasonable.

The prevalence of $\mathrm{HU}$ increased dramatically in female subjects aged between 40 (0.9\%) and $50(1.7 \%)$ years in accordance with a previous study in Japan $(1.3 \%$ and $3.7 \%$ in the age group of under 50 years, and more than or equal to 50 years, respectively). ${ }^{13}$ This substantial difference in the prevalence of $\mathrm{HU}$ between the ages of 40 and 50 years was also observed in the Third US National Health and Nutritional Examination Survey (NHANES III, 1988 to 1994$)$ study. ${ }^{10}$ It is considered that this exponential increase of the prevalence is caused by menopause because estrogen enhances the renal urate clearance. ${ }^{19}$ The increased use of diuretics in association with increasing age is also considered to be one of the explanations for the exponential increase in prevalence because diuretics are a well-known cause of HU. ${ }^{20}$

Although an increasing trend in the prevalence of $\mathrm{HU}$ and age specific prevalence over the five-year period from FY 2010 to FY 2014 were not observed either in male or female subjects (Figure 2a-c) in this study, a previous study 
using the health check-up data reported that the prevalence of $\mathrm{HU}$ in Japanese male subjects showed an increasing trend in all age groups from 1996 to $2004 .{ }^{12}$ This changing trend in the prevalence of $\mathrm{HU}$ suggests changes in the prevalence of the risk factors of $\mathrm{HU}$ between the 1990s to 2000s and 2010s. According to the results of the National Health and Nutrition Survey in Japan, there was an increasing trend in the prevalence of obesity (BMI $\geq 25$ ) in male subjects in the 1990s to 2000s (22.0\% in 1996 and $27.3 \%$ in 2004), while the prevalence has not changed in recent years $(29.3 \%$ in 2010 and $27.8 \%$ in 2014) without big change in blood pressure, triglyceride, blood glucose or total cholesterol during the period. ${ }^{21}$ Although other risk factors of $\mathrm{HU}$ such as CKD may influence the prevalence of $\mathrm{HU},{ }^{22}$ it is considered that obesity or BMI was one of the major factors that triggered the changing trend in the prevalence of HU.

It is well known that SUA is associated with the prevalence of metabolic syndrome and its components. ${ }^{23-25}$ In this study, we conducted subgroup analyses by the presence or absence of well-known comorbidities, BMI category, and smoking status, to investigate the consistency between previous studies and this study regarding the association of these cardiovascular risk factors with the prevalence of $\mathrm{HU}$. As a result, the positive associations of $\mathrm{HU}$ with its comorbidities, BMI and smoking status were confirmed with the exception of DM and smoking status in male subjects.

There is much evidence that indicates a relationship between $\mathrm{HU}$ and $\mathrm{HT}^{2,26}$ as well as $\mathrm{HU}$ and HL. ${ }^{24,27}$ Obesity is also a well-known risk factor of $\mathrm{HU}$, and several studies have reported a relationship between $\mathrm{HU}$ and BMI. ${ }^{28-30}$ This study showed that the prevalence of $\mathrm{HU}$ in male and female subjects who had HT or HL was higher than that in subjects who did not have HT or HL, as well as a clear positive association between $\mathrm{BMI}$ and the prevalence of $\mathrm{HU}$ (Figure $3 \mathrm{a}$ and $3 \mathrm{~b}$ ), which were consistent with the results of previous studies.

In regard to the association between $\mathrm{HU}$ and $\mathrm{DM}$, sex differences in the prevalence of $\mathrm{HU}$ between DM and non-DM subjects were observed in this study (Figure $3 \mathrm{a}$ and $3 \mathrm{~b}$ ). Interestingly, a negative association between $\mathrm{HU}$ and the occurrence of DM in males, ${ }^{27,31}$ and difference between sexes with regards to this association have been reported previously. ${ }^{28,32}$ There is evidence that mean serum urate increases with increasing glucose concentration up to $7.0 \mathrm{mmol} / \mathrm{L}$ in males and $9.0 \mathrm{mmol} / \mathrm{L}$ in females, and thereafter increasing glucose values are accompanied by a decrease in serum urate. ${ }^{33}$ This difference in the flexion point of the glucose concentration between the sexes may be one of the explanations of the sex differences in the prevalence of $\mathrm{HU}$ between DM and non-DM subjects, observed in this study. However, further research is necessary to elucidate the reason of the sex differences in the relationship between $\mathrm{HU}$ and DM.

As for the relationship between smoking and $\mathrm{HU}$, an inverse association between smoking and SUA levels has been described. ${ }^{34,35}$ In accordance with these findings, male subjects with a smoking habit showed a lower prevalence of $\mathrm{HU}$ (Figure 3a). On the other hand, smoking habit was associated with a higher prevalence of $\mathrm{HU}$ in female subjects (Figure 3b). However, to our knowledge, the difference in the effect of smoking on SUA levels by sex has never been reported before. The reason for the negative correlation between SUA levels and smoking is partially explained as a reduced production and increased consumption of endogenous antioxidant uric acid caused by cigarette smoking, which is a source of oxidative stress. ${ }^{34}$ Further research is needed to understand the exact mechanism of this association.

Several limitations due to the characteristics of health insurance claims data, health check-up data, and the method of analysis should be considered in this study. Since the MinaCare database includes healthcare information of working individuals and their dependents, it may not cover general retired people. Therefore, generalizability of the prevalence in subjects aged 65 years and older is possibly lower than that in other age categories. It is also considered that the precision of the prevalence in subjects aged under 20 years and those aged 65 years and older is lower than that in other age categories due to the small sample sizes. However, it is reported that age trends by sex for the parameters such as blood pressure, lipid parameters and blood glucose levels were generally consistent across the MinaCare database and national survey data sources. ${ }^{14}$ In addition, since $\mathrm{HU}$ is not a 
life-threatening disease, it is considered that most $\mathrm{HU}$ patients can continue working. Therefore, there are no major differences between working individuals and the general population in terms of the distribution of SUA levels in the same age group and the generalizability of the estimated prevalence of $\mathrm{HU}$ is considered sufficiently high, particularly in young and middle-aged people.

There is another limitation associated with the health check-up data, particularly the arbitrary property of SUA measurements of health check-ups. We cannot completely deny the possibility of selection bias because we included those who had at least one SUA measurement of health check-up data as the study subjects. However, the characteristics of this study population are similar to those in a previous study using the same database, which included those who had at least one set of health check-up data as the study subjects. ${ }^{14}$ This suggests that the existence or non-existence of SUA measurements do not depend on the individual SUA levels, but depend on the health insurance to which insured persons belong. Therefore, it is considered that selection bias caused by the extraction of the study population is not major in this study.

Some limitations based on the analysis method should also be considered. We only performed univariate analyses and did not adjust for other risk factors in the analysis that investigated the association between the prevalence of $\mathrm{HU}$ and its comorbidities, because these subgroup analyses were exploratory ones. Since the major comorbidities of HU such as HT, DM, HL, and obesity are associated with each other, we cannot deny overestimation of the strength of association between $\mathrm{HU}$ and these comorbidities.

In addition to this, other limitations derived from the definition of $\mathrm{HU}$ are conceivable. We defined HU patients as people to whom ULDs were prescribed in this study, while there are some anti-hypertensive and anti-hyperlipidemic drugs that have a moderate SUA-lowering effect. Therefore, if these drugs were intentionally prescribed to HT or HL patients with a high SUA level, we cannot deny the possibility of underestimation of $\mathrm{HU}$ prevalence due to a lack of coverage of the potential HU patients whose SUA levels were controlled at $7.0 \mathrm{mg} / \mathrm{dL}$ or below by the administration of these drugs.
Finally, similar to studies using administrative or claims databases, there are several limitations that may affect the validity and reproducibility of the results such as incomplete coding, coding errors, and correction or duplication of claims.

The strengths of this study are that the MinaCare database, which includes linked health check-up data and claims data, enabled us to estimate the prevalence of $\mathrm{HU}$ in the Japanese population that also includes well-managed HU patients, and the sample size of this study was also larger than those of previous studies.

In conclusion, this is the first study using a Japanese healthcare database to estimate the prevalence of $\mathrm{HU}$ in Japan considering both SUA levels and medication status. The increment in prevalence reported by this study compared with those in previous studies may reflect the number of well-managed $\mathrm{HU}$ patients whose SUA levels were controlled at $7.0 \mathrm{mg} / \mathrm{dL}$ or below by the administration of ULDs. The fact that medication with ULDs in males increased dramatically with age supports the hypothesis of the underestimation of the previously reported prevalence in older male patients stated in the Japanese guideline for the management of hyperuricemia and gout. Considering the administration of ULDs, the prevalence of $\mathrm{HU}$ in the Japanese population increased with age in this study. These results suggest that the number of HU patients in Japan is higher than had previously been assumed. In addition, the association between $\mathrm{HU}$ and its comorbidities which are also major cardiovascular risk factors were confirmed in this study. Therefore, it is important to increase awareness on $\mathrm{HU}$ in society to reduce the social and health burden of HU-related diseases.

\section{Acknowledgements}

The authors would like to thank Shin Fujimori of Teikyo University Shinjuku Clinic for his helpful advice.

\section{Declaration of conflicting interests}

SH, MY, DS, YI, and YF are full-time employees of Pfizer Japan Inc. YY is the founder and CEO of MinaCare Co., Ltd., and SK is full-time employee of MinaCare Co., Ltd.

\section{Funding}

This research was sponsored and funded by Pfizer. 


\section{REFERENCES}

1. Campion EW, Glynn RJ, DeLabry LO. Asymptomatic hyperuricemia. Risks and consequences in the Normative Aging Study. Am J Med 1987;82:421-6.

2. Wang J, Qin T, Chen J, Li Y, Wang L, Huang H, et al. Hyperuricemia and risk of incident hypertension: a systematic review and meta-analysis of observational studies. PLoS One 2014;9:e114259.

3. Grayson PC, Kim SY, LaValley M, Choi HK. Hyperuricemia and incident hypertension: a systematic review and meta-analysis. Arthritis Care Res (Hoboken) 2011;63:102-10.

4. Zhu P, Liu Y, Han L, Xu G, Ran JM. Serum uric acid is associated with incident chronic kidney disease in middle-aged populations: a meta-analysis of 15 cohort studies. PLoS One 2014;9:e100801.

5. Hsu CY, Iribarren C, McCulloch CE, Darbinian J, Go AS. Risk factors for end-stage renal disease: 25-year follow-up. Arch Intern Med 2009;169:342-50.

6. Billiet L, Doaty S, Katz JD, Velasquez MT. Review of hyperuricemia as new marker for metabolic syndrome. ISRN Rheumatol 2014;2014:852954.

7. Eckel RH, Grundy SM, Zimmet PZ. The metabolic syndrome. Lancet 2005;365:1415-28.

8. Fujimori $\mathrm{S}$, Itoh $\mathrm{H}$, Kato $\mathrm{K}$, Watanabe $\mathrm{H}$, Matsuura $\mathrm{H}$, Ogata $\mathrm{N}$, et al. The frequencies of hyperuricemia and gout may not increase in Japan. Gout and Nucleic Acid Metabolism 2006;30:13-20.

9. Kamei K, Konta T, Ichikawa K, Sato H, Suzuki N, Kabasawa A, et al. Serum uric acid levels and mortality in the Japanese population: the Yamagata (Takahata) study. Clin Exp Nephrol 2016;20:904-9.

10. Rho YH, Zhu Y, Choi HK. The epidemiology of uric acid and fructose. Semin Nephrol 2011;31:410-9.

11. Liu B, Wang T, Zhao HN, Yue WW, Yu HP, Liu CX, et al. The prevalence of hyperuricemia in China: a meta-analysis. BMC Public Health 2011:11:832.

12. Tomita M, Mizuno S. Is the number of patients with hyperuricemia increasing? - Focusing on gender differences. Gout Nucleic Acid Metab 2006;30:1-5.

13. Japanese Society of Gout and Nucleic Acid Metabolism Guideline Revisory Committee, ed. Revised Guideline for the Management of Hyperuricemia and Gout. Osaka: Medical Review Co. Ltd; 2010. p. 32-4.

14. Shima D, Ii Y, Yamamoto Y, Nagayasu S, Ikeda Y, Fujimoto Y. A retrospective, cross-sectional study of real-world values of cardiovascular risk factors using a healthcare database in Japan. BMC Cardiovasc Disord 2014;14:120.

15. The Ministry of Education, Culture, Sports, Science and Technology of Japan, the Ministry of Health, Labour and Welfare of Japan. Ethical Guidelines for Medical and Health Research Involving Human Subjects. Available from: http://www.mhlw.go.jp/ file/06-Seisakujouhou-10600000-Daijinkanbouko useikagakuka/0000080278.pdf [Accessed Apr 18, 2017].
16. Clopper CJ, Pearson ES. The use of confidence or fiducial limits illustrated in the case of the binomial. Biometrika 1934;26:404-13.

17. Feng Y, Jiang T, Wang L. Hyperuricemia and acute kidney injury secondary to spontaneous tumor lysis syndrome in low risk myelodysplastic syndrome. BMC Nephrol 2014;15:164.

18. Kuo CF, Grainge MJ, Zhang W, Doherty M. Global epidemiology of gout: prevalence, incidence and risk factors. Nat Rev Rheumatol 2015;11:649-62.

19. Barr WG. Uric acid. In: Walker HK, Hall WD, Hurst JW, editors. Clinical Methods: The History, Physical, and Laboratory Examinations. 3rd ed. Boston: Butterworths; 1990. p. 770-2.

20. Palmer BF. Metabolic complications associated with use of diuretics. Semin Nephrol 2011;31:542-52.

21. The Ministry of Health, Labour and Welfare of Japan. National Health and Nutrition Survey data. Available from: http://www.mhlw.go.jp/seisakunitsuite/ bunya/kenkou_iryou/kenkou/kenkounippon21/en/ eiyouchousa/keinen_henka_shintai.html. [Accessed: April 18, 2017].

22. Gustafsson D, Unwin R. The pathophysiology of hyperuricaemia and its possible relationship to cardiovascular disease, morbidity and mortality. BMC Nephrol 2013;14:164.

23. Hjortnaes J, Algra A, Olijhoek J, Huisman M, Jacobs $J$, van der Graaf Y, et al. Serum uric acid levels and risk for vascular diseases in patients with metabolic syndrome. J Rheumatol 2007;34:1882-7.

24. Babio N, Martínez-González MA, Estruch R, Wärnberg $\mathrm{J}$, Recondo $\mathrm{J}$, Ortega-Calvo $\mathrm{M}$, et al. Associations between serum uric acid concentrations and metabolic syndrome and its components in the PREDIMED study. Nutr Metab Cardiovasc Dis 2015;25:173-80.

25. Norvik JV, Storhaug HM, Ytrehus K, Jenssen TG, Zykova SN, Eriksen BO, et al. Overweight modifies the longitudinal association between uric acid and some components of the metabolic syndrome: The Tromsø Study. BMC Cardiovasc Disord 2016;16:85.

26. Kuwabara M, Niwa K, Nishi Y, Mizuno A, Asano T, Masuda K, et al. Relationship between serum uric acid levels and hypertension among Japanese individuals not treated for hyperuricemia and hypertension. Hypertens Res 2014;37:785-9.

27. Kono S, Shinchi K, Imanishi K, Honjo S, Todoroki I. Behavioural and biological correlates of serum uric acid: a study of self-defence officials in Japan. Int $J$ Epidemiol 1994;23:517-22.

28. Nagahama K, Iseki K, Inoue T, Touma T, Ikemiya $Y$, Takishita S. Hyperuricemia and cardiovascular risk factor clustering in a screened cohort in Okinawa, Japan. Hypertens Res 2004;27:227-33.

29. Nakanishi N, Tatara K, Nakamura K, Suzuki K. Risk factors for the incidence of hyperuricaemia: a 6-year longitudinal study of middle-aged Japanese men. Int $J$ Epidemiol 1999;28:888-93. 
30. Wang H, Wang L, Xie R, Dai W, Gao C, Shen P, et al. Association of serum uric acid with body mass index: A cross-sectional study from jiangsu province, China. Iran J Public Health 2014;43:1503-9.

31. Herman JB, Goldbourt U. Uric acid and diabetes: observations in a population study. Lancet 1982;2:240-3.

32. Fang J, Alderman MH. Serum uric acid and cardiovascular mortality the NHANES I epidemiologic follow-up study, 1971-1992. National Health and Nutrition Examination Survey.
JAMA 2000;283:2404-10.

33. Whitehead TP, Jungner I, Robinson D, Kolar W, Pearl A, Hale A. Serum urate, serum glucose and diabetes. Ann Clin Biochem 1992;29:159-61.

34. Hanna BE, Hamed JM, Touhala LM. Serum uric Acid in smokers. Oman Med J 2008;23:269-74.

35. Haj Mouhamed D, Ezzaher A, Neffati F, Douki W, Gaha L, Najjar MF. Effect of cigarette smoking on plasma uric acid concentrations. Environ Health Prev Med 2011;16:307-12. 